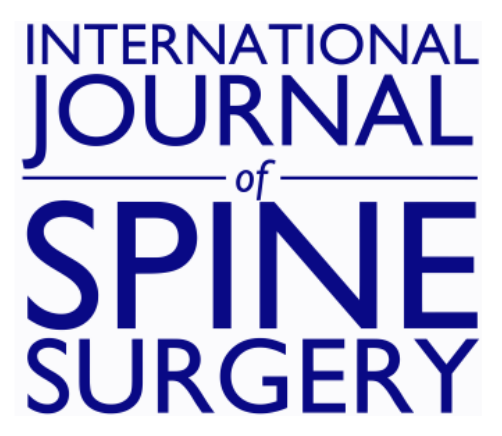

\title{
The Learning Curve in Foraminal Endoscopic Discectomy: Experience Needed to Achieve a 90\% Success Rate
}

\author{
Rudolf Morgenstern, Christian Morgenstern and Anthony T. Yeung
}

Int J Spine Surg 2007, 1 (3) 100-107

doi: https://doi.org/10.1016/SASJ-2007-0005-RR

http://ijssurgery.com/content/1/3/100

This information is current as of April 26, 2023.

Email Alerts Receive free email-alerts when new articles cite this article. Sign up at:

http://ijssurgery.com/alerts 


\title{
The Learning Curve in Foraminal Endoscopic Discectomy: Experience Needed to Achieve a $\mathbf{9 0 \%}$ Success Rate
}

Rudolf Morgenstern, MD, PhD, Christian Morgenstern, Dipl-Ing, and Anthony T. Yeung, MD

\begin{abstract}
Background

We sought to construct a general methodology for objectively quantifying the learning curve associated with any surgical technique and to determine the number of cases needed to achieve a success rate of $90 \%$ for the technique of transforaminal endoscopic lumbar discectomy. To our knowledge, no other studies have observed the learning curve of endoscopic lumbar discectomy by transforaminal approach.

Methods

We studied the learning curve of 1 orthopedic surgeon who had had experience performing open spine surgery and knee and shoulder arthroscopic surgery, but not endoscopic spine surgery. We studied 144 patients who had an endoscopic lumbar discectomy by transforaminal approach (using the Yeung Endoscopic Surgery System). We evaluated results with modified MacNab criteria and used a questionnaire to determine the patients' satisfaction with the surgery. The average follow-up period was 24 months. We used an algorithm, analyzing the patient outcome and the surgical time evolution, to determine the case at which a success rate of $90 \%$ good/excellent results was reached.
\end{abstract}

Results

The cut for the calculated learning curve was placed at case no. 72; i.e., the results in the first 72 cases were $75 \%$ good/excellent, $18 \%$ fair, and $7 \%$ poor, and the results in the following 72 cases were $90.3 \%$ good/excellent, $9.7 \%$ fair, and $0 \%$ poor.

Conclusions

A methodology to calculate the learning curve of a surgical procedure was developed. A learning curve of 72 cases was needed to reach the goal of $90 \%$ of good/excellent results for transforaminal endoscopic lumbar discectomy.

\section{Clinical Relevance}

The method developed to establish the learning curve of a surgical procedure, based on outcome and surgical time, may be used to assess any new procedure. With respect to the transforaminal endoscopic technique, the determination of a specific number of cases (72) needed to master (achieve $90 \%$ excellent/ good results) could help orient surgeons willing to adopt this technique.

Key Words Foraminal endoscopic lumbar discectomy, learning curve, transforaminal approach. SAS Journal. Summer 2007;1:100-107. DOI: SASJ-2007-0005-RR

\section{INTRODUCTION}

Endoscopic transforaminal decompression and fragmentectomy techniques for lumbar disc herniation is evolving as a minimally invasive technique. This approach has evolved to allow access to the pathoanatomy of many painful degenerative conditions of the lumbar spine with low surgical morbidity. ${ }^{1-4}$ However, the technique remains foreign to most spine surgeons. There is a perception that the learning curve is insurmountable for most surgeons even if the proponent surgeon and the technique gain the acceptance of his or her peers. Surgeons who take the time to learn, have specific training in their fellowships, or have actually scrubbed in with mentor surgeons may find the learning curve much less steep. The high learning curve, without a long hands-on training period, discourages most surgeons from adopting the endoscopic technique.

We present, first, a generalized methodology to objectively quantify the learning curve of any surgical procedure. Then, we describe how the presented methodology was applied to quantify the learning curve projected for R.M., an orthopedic 
surgeon with 10 years of joint arthroscopy and 8 years of traditional spine surgery experience, to achieve the technical ability to treat a wide spectrum of contained and extruded herniated disc fragments that match the results published by his mentor, A.Y. ${ }^{1-3}$ R.M. learned the endoscopic surgical technique in several intensive training workshops at A.Y.'s clinic (Squaw Peak Surgical Facility, Phoenix, Arizona) and under his direct supervision. R.M. adopted the endoscopic YESS technique and practiced it as described and taught by A.Y.

During the 5 years of the study, R.M. attended at least 1 endoscopic workshop with cadaver training in the United States per year.

The first posterolateral discectomy was a percutaneous central nuclectomy $^{5}$ in 1975, followed by Kambin and Gellman's ${ }^{6}$ report of 9 cases in 1983. In 1983 Forst and Hausmann ${ }^{7}$ reported the direct visualization of intervertebral disc space with a modified arthroscope. Schreiber et al. ${ }^{8}$ used a biportal endoscopic technique. The transforaminal approach was reported by Mathews ${ }^{9}$ in 1996 , but this approach is the same portal utilized by Kambin ${ }^{6}$ and Yeung. ${ }^{3}$ To aid visualization, Yeung ${ }^{1-3}$ utilized laser as an adjunct to endoscopic discectomy. Yeung ${ }^{1}$ and Knight $^{10}$ later used the holmium: yttriumaluminum-garnet (Ho:YAG) laser for foraminoplasty and decompression of stenosis. In 1997 Yeung $^{1-3}$ introduced a rigid rod-lens, flow-integrated, and multichannel operating spinal endoscope with slotted and bevel-ended cannulas that allowed for same-field viewing of the epidural space, annular wall, and intradiscal space.

\section{MATERIALS AND METHODS}

From January 2001 to June 2005, R.M. performed posterolateral endoscopic excisions of lumbar disc herniation, L1-L2 to L5S1, on 144 consecutive patients with the Yeung Endoscopic Surgery System (YESS) technique. ${ }^{1-3}$

The general inclusion criteria were clinical evidence of lumbar disc herniation and findings from a physical examination consistent with the magnetic resonance imaging (MRI) findings. Every patient had had at least 3 months of failed nonsurgical treatment and clinical signs of radiculopathy that included intractable leg or buttock pain with or without back pain. Lumbar sagittal and frontal x-rays and MRI were the standard minimal images used to correlate symptoms of back and neuropathic pain. All 144 patients underwent discography to visualize disc degeneration and stain degenerative nucleus pulposus for surgical removal and to correlate the reproduction of concordant pain. ${ }^{3}$ To perform an endoscopic transforaminal approach, it is necessary to first insert a needle into the disc. The addition of discography as a complementary step provides additional information to confirm that the disc is painful under increased internal pressure. It also helps to verify the herniation shape and stains the degenerative nucleus pulposus blue with a vital dye (indigo carmine) for targeted disc extraction. The entire procedure is performed under local anaesthesia and light patient sedation, so that the patient is able to respond to simple verbal orders and react to pain stimuli.

To be considered positive for the endoscopic procedure, the discogram pattern has to show an abnormal pattern (contrast escapes or profiles herniation) and positive pain production. Discographic exclusion criteria are normal disc shape and nonreproduction of concordant pain. The transforaminal endoscopic procedure was performed only at levels found to be positive as determined by concordant pain reproduction and an abnormal discogram pattern. The procedure was performed as described by Yeung ${ }^{1-3}$ using a $20^{\circ}$ rigid endoscope with a working channel of $2.8 \mathrm{~mm}$ (YESS; Richard Wolf GmbH, Knittlingen, Germany); Laser Ho:YAG 80 Watt with $90^{\circ}$ side firing electrodes (Trimedyne Inc, Irvine, California); radiofrequency coagulation system (Ellman International Inc, Hewlett, New York); and indigo carmine (Taylor Pharmaceuticals, Decatur, Illinois) diluted with iopamidol 300 1:10 to blue stain abnormal nucleus pulpous and annular fissures. ${ }^{8}$

In accordance with the procedure, after the needle insertion and the discogram, a dilator is passed using the needle central guide. This central guide is then extracted and a $30^{\circ}$ bevelled cannula is passed over the dilator and the dilator extracted. The fluoroscopy X-ray arch is used to control in anterior-posterior and lateral view the proper position of the dilator and cannula into the disc through the foraminal approach. Then the endoscope is passed through the cannula and, under saline irrigation, the disc structures are visible on the camera monitor.

The careful dissection of the ligament and disc tissues with laser energy and single-action basquets allows the surgeon to see the blue-stained nucleus pulposus and the herniation and, with careful identification, the neural structures. A foraminoplasty can be performed with the laser to ablate the upper part of the inferior facet and the articular capsule. Sometimes that foraminoplasty could be essential to arrive at the herniation, especially at the L5-S1 level and for caudal migrated herniations. After the herniation removal and a disc curettage of the remaining nuclear loose or degenerated fragments, the endoscope can be removed and the skin sutured $(5 \mathrm{~mm})$. A corticoid such as Depomedrol $125 \mathrm{mg}$ is locally injected before the skin suture.

Every procedure was video-recorded (with miniDV) for subsequent analysis. Discography images were printed and added to the patient's documentation.

\section{STATISTICAL METHODS}

A relational database and client software was specifically designed by C.M. (BDM version 2.1) to allow storage of the patients' personal data and the cases' documentation. The software calculates follow-up median and standard deviation, age median and standard deviation, and the outcome distribution of the operations by sex. 
We obtained the follow-up data for each patient by calculating the difference in days between the date of the operation and the ending date of our study. We then computed the overall median and standard deviation for the follow-up data. We converted the days into months by dividing by 30 .

The operated disc levels and the type of herniations can be seen in Table 1. There were 96 (66.7\%) male patients and $48(33.3 \%)$ female patients. The average male patient's age was 45.9 years and the average female patient's age was 44.6 years. The age range was 18 to 76 years. Global age average was 45.5 years; standard deviation was 12.42 years.

Table 1

Case Distribution of Operated Disc Levels and Herniation Types/Locations $(n=204)$

\begin{tabular}{cc}
\hline & No. (\%) \\
\hline Disc level & $3 \quad(1.5)$ \\
L1-L2 & $7 \quad(3.4)$ \\
L2-L3 & $25(12.3)$ \\
L3-L4 & $92(45.0)$ \\
L4-L5 & $77(37.8)$ \\
L5-S1 & \\
Herniation type/location & $60(29.4)$ \\
Bulging & $26(12.8)$ \\
Central & $46(22.6)$ \\
Lateral & $71(34.7)$ \\
Foraminal & $1(0.5)$ \\
Extraforaminal & \\
\hline
\end{tabular}

\section{Parameters}

The first parameter for determining the learning curve was evaluation of the success of the surgical procedure on the basis of the patient's clinical record and a questionnaire of 4 yes/no questions, as described by Yeung and Tsou, ${ }^{1,2}$ administered a minimum of 6 months after the surgical procedure:

1) Since your endoscopic spine surgery, have you had subsequent lumbar spine surgery at the same level?

2) Are you satisfied with the outcome of your endoscopic operation?

3) Would you select the same endoscopic spine surgery again in the future, given the same disc herniation and your personal familiarity with the operative experience?

4) Are your current back or leg symptoms, if any, worse than before your endoscopic back surgery?
If 1 answer deviated from the pattern of "no, yes, yes, no," we considered the surgical procedure to have failed. The results were classified with the modified MacNab criteria shown in Table 2. We chose to use MacNab criteria because they have been used in other published, peer-reviewed outcome studies of the YESS technique. ${ }^{1,2}$

\section{Table 2}

MacNab Criteria

\begin{tabular}{|c|c|}
\hline Result & Criteria \\
\hline Excellent & Patient is asymptomatic; medication is not required. \\
\hline Good & $\begin{array}{l}\text { Patient recovers fully from sciatica symptoms but requires } \\
\text { occasional medication for residual or recurrent pain. }\end{array}$ \\
\hline Fair & Patient recovers partially and requires regular medication. \\
\hline Poor & $\begin{array}{l}\text { Patient does not improve or recover or recovers only par- } \\
\text { tially, requiring medication on a regular basis; patient not } \\
\text { satisfied with the surgical results. }\end{array}$ \\
\hline
\end{tabular}

The second parameter for the determination of the learning curve was analysis of the evolution of the surgical time until stabilization. ${ }^{11}$ We measured surgical time as the elapsed time between the first needle skin puncture and the final skin suture. Preoperational instrument preparation and anesthesia procedures are excluded from this time measurement.

Surgical time is a general but not determining parameter that helps approximate the cutpoint, i.e., the point at which good and excellent results make up $90 \%$ of all results. The parameter of patient outcome contains information about the final results and will therefore weigh more in determining the cutpoint.

\section{Learning Curve}

As described by Cook et al., ${ }^{11}$ a learning curve must contain a starting point (normally the first case), a learning rate (with increasing performance on patient outcome), and an asymptote when the expert level (here, 90\%) is reached.

We designed an algorithm to analyze the patient outcome and to determine the case number at which the "expert" rate of $90 \%$ of successful results was reached. We chose this rate of success, because the rate accepted as equivalent to the inventor's technique in the literature is $91.2 \%{ }^{1,2}$ :

$$
\text { (1) } Y=100 \times \frac{[N-M(N)]}{N}
$$

where $Y=\%$ successful results for $N$ cases, $N=$ number of cases (here, 1-144), and $M(N)=$ the sum of fair and poor cases within $N$ cases.

$N$, as seen in equation 1 , can be interpreted as a cutpoint within the total number of cases, $T=144$. The algorithm runs 
iteratively for $N=1,2,3 \ldots T$, calculating for each $N$ the success rate $Y_{\text {Res }}$ (see equation 2 ) of all the cases following the dividing point $N$, while ignoring the cases previous to $N$ and $N$ itself.

$$
\text { (2) } Y_{\operatorname{Re} s}(N)=1-\frac{M_{\operatorname{Re} s}}{N_{\operatorname{Re} s}}
$$

where $M_{\text {Res }}=$ the sum of fair and poor cases after $N, N_{\text {Res }}=$ the sum of all cases after $N$, and $Y_{\text {Res }}=$ success rate as a percentage.

\section{RESULTS}

The obtained raw curve for the patient outcome (ordinate $Y_{\mathrm{Res}}$, abscissa $N$ ) has been smoothed by interpolation with a Splines algorithm (MATLAB version 6.1; The Mathworks Inc., Natick, Massachusetts) and is represented in Figure 1.

\section{Figure 1}

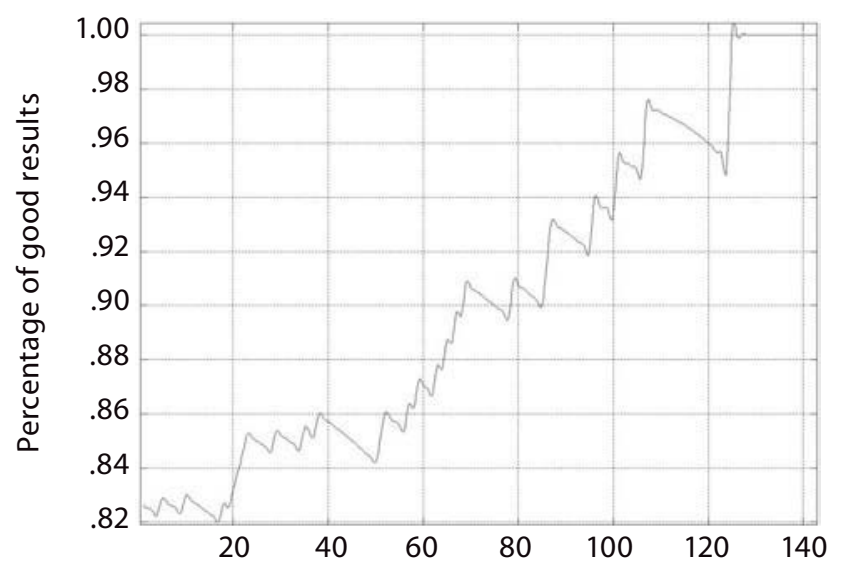

Number of cases
Figure 2

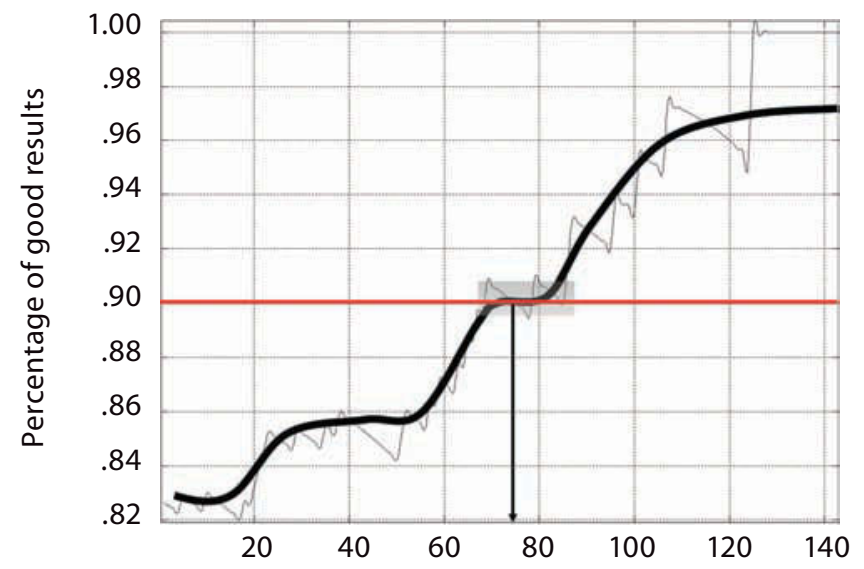

Number of cases

Averaged learning curve (black line) marked with $90 \%$ good/excellent cutpoint (red line).

Figure 3

In Figure 2 the learning curve has been averaged (black line) and the shaded box (cases 67-85) is placed where the learning curve oscillates around the $90 \%$-successful line. Any case taken from this area could represent the end of the learning curve.

The surgical time evolution, as described by Cook et al., ${ }^{11}$ is represented in Figure 3. To minimize the influence of the number of operated discs and the difficulty of every single case, we averaged the surgical time every 20 cases.

The optimal surgical time, as determined by Yeung et al. ${ }^{1}$ and Tsou et al., ${ }^{2}$ was 45 minutes, so the control time ${ }^{11}$ was also 45 minutes. As seen in Figure 3, the surgical time asymptote is placed after case no. 80, so that the optimal operation time reached by R.M. for this limited number of cases was approximately 50 minutes.

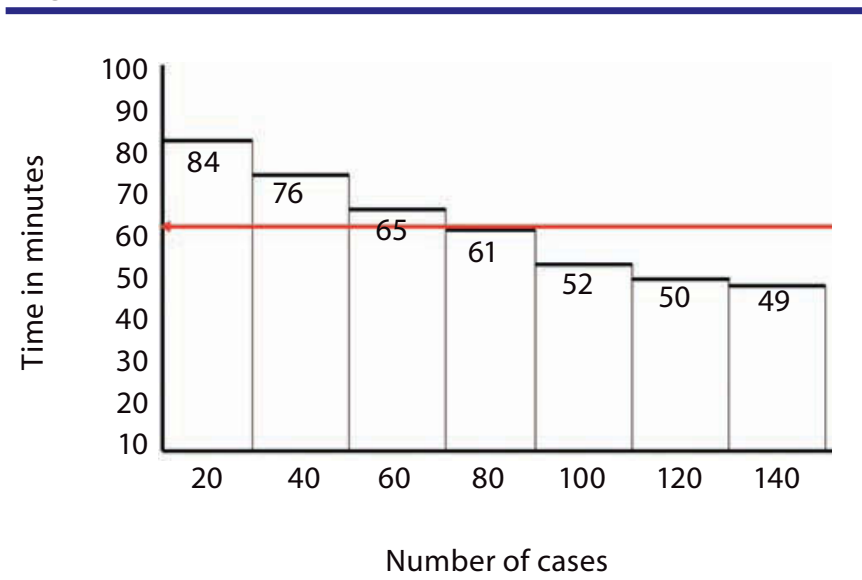

Number of cases

Evolution of required surgical time (averaged every 20 cases).

In Figure 4, the learning curve of patient outcome (Figure 2) has been superimposed on the averaged surgical time steps (Figure 3). The optimal surgical time in the literature ${ }^{1,2}$ is 45 minutes for 1 single-disc operation with a $90 \%$ expectation of good and/or excellent results. Because the surgical time in this study was calculated for an average of 1.41 discs per case, the time scale was adjusted from 45 minutes for 1 disc to 63 minutes and 27 seconds for 1.41 disc per operation.

In Figure 4, the average surgical time meets the learning curve around the line for $90 \%$ of results being successful. This area contains case no. 72. The approximation using both parameters places the critical case number within the area after which the average surgical time stabilization begins. Case no. 72 can be 


\section{Figure 4}

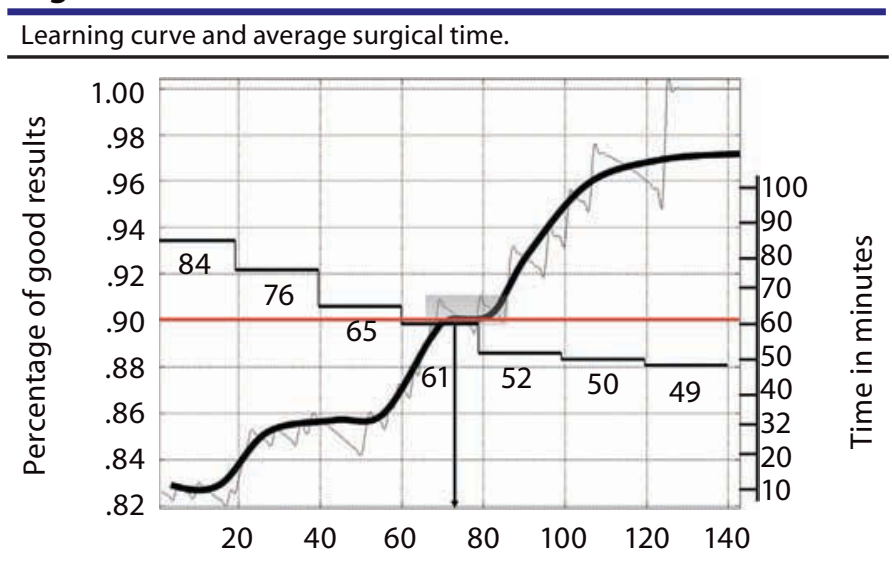

Number of cases

Average surgical time (represented by a line of steps) was calculated every 20 cases.

taken as a good approximation for the end of the learning effect (cases 67-85), as it is shown by both curves, and furthermore splits the 144 cases into 2 groups with the same number of samples.

The overall results, using MacNab criteria, can be found in Table 3 and are graphically represented in Figure 5. Physical therapists involved with the patients' postoperative care independently evaluated the results; questionnaires remained anonymous. (These therapists are independent professionals who routinely participate in the rehabilitation of patients of R.M.) The clinical record or the feedback on the questionnaire indicated fair or poor results for 25 out of 144 patients for the following reasons:

Table 3

Overall Results of Foraminal Endoscopic Discectomy $(n=144)$

\begin{tabular}{lr} 
Result & No (\%) \\
\hline Excellent/good & $119(82.6)$ \\
Fair & $20(13.9)$ \\
Poor & $5(3.5)$
\end{tabular}

- Postoperative neuropathic pain (11 cases). Ten of the neuritis cases were temporary dysesthesias. Neuropathic pain is described as neuropathy following a nerve root pattern that started postoperatively or within 4 days of surgery and required medication for more than 1 week but less than 3 months. One patient with dropfoot syndrome has recovered partially. This patient was rated as having a poor result.
Figure 5

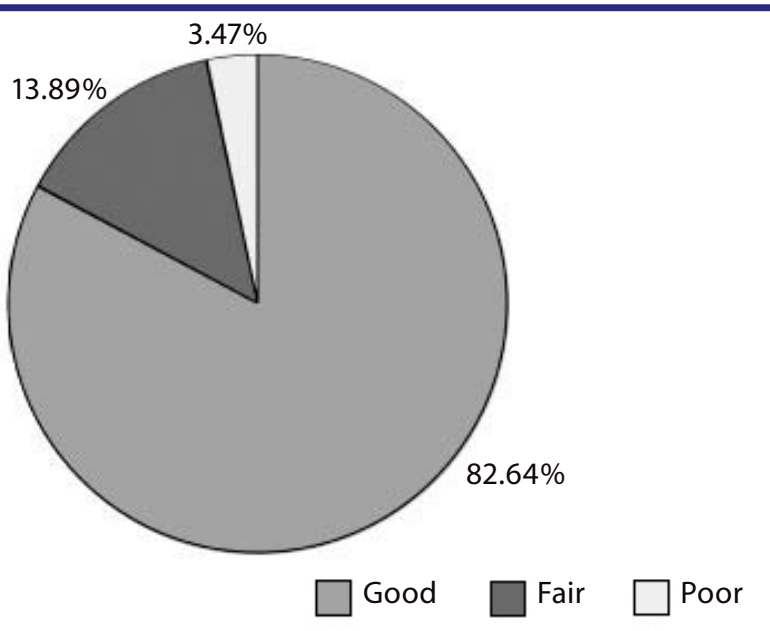

Overall results of the 144 cases.

- Reinterventions caused by foraminal stenosis or residual fragments missed at surgery (8 cases). Five were open reinterventions and 3 were endoscopic reinterventions. Whether the fragment represented residual or recurrent herniation was not always clear, but it was deemed residual if sciatica was not completely resolved postoperatively.

- Sterile discitis (2 cases). Origin unknown.

- Underestimated spinal stenosis (1 case).

- Instabilities underestimated at patient selection (3 cases).

At least one half of the surgical failures can be attributed to the surgeon's learning curve in recognizing and the technical ability to treat the pathoanatomy of the patient's pain.

Of the 144 cases, 90 patients underwent rehabilitation under the direct supervision of R.M. The rehabilitation was based on electrotherapy (transcutaneous electrical nerve stimulation) and myorelaxation during the immediate postoperative period, and lumbar stabilization plus muscular reconditioning training up to 12 weeks after surgery. ${ }^{12}$ Forty-three patients were treated in other locations but maintained contact with R.M. at least every 3 months for a neurological examination and a personal interview about pain evolution and work ability.

For 11 cases, feedback on rehabilitation was lacking. From these 11 cases, 7 were considered as failed surgery, because the patients answered the questionnaire in a negative way or did not answer it at all. For the remaining 4 cases, the patients answered the questionnaire in a way that indicated positive results.

The overall follow-up rate was $92 \%$. The average followup period was 24 months with a standard deviation of 13 
months. As stated earlier, an independent group of professional physiotherapists collected, evaluated, and analyzed the followup data and the questionnaire for every patient. ${ }^{12}$

As explained, the authors split the results of the 144 patients around case no. 72, resulting in 2 groups of 72 cases each. The $\mathrm{MacNab}$ results for group 1 (cases 1-72) and for group 2 (cases 73-144) can be found in Table 4. The distribution of results for both groups is represented in Figure 6.

\section{Table 4}

Results for Group 1 (Cases 1-72; $n=72$ ) and Group 2 (Cases 73-144; $n=72$ ) Receiving Foraminal Endoscopic Discectomy

\begin{tabular}{lcc} 
Result & Group 1, No. (\%) & Group 2, No. (\%) \\
\hline Excellent and good & $54(75.0)$ & $65(90.3)$ \\
Fair & $13(18.0)$ & $7(9.7)$ \\
Poor & $5(7.0)$ & $0(0.0)$
\end{tabular}

Figure 6

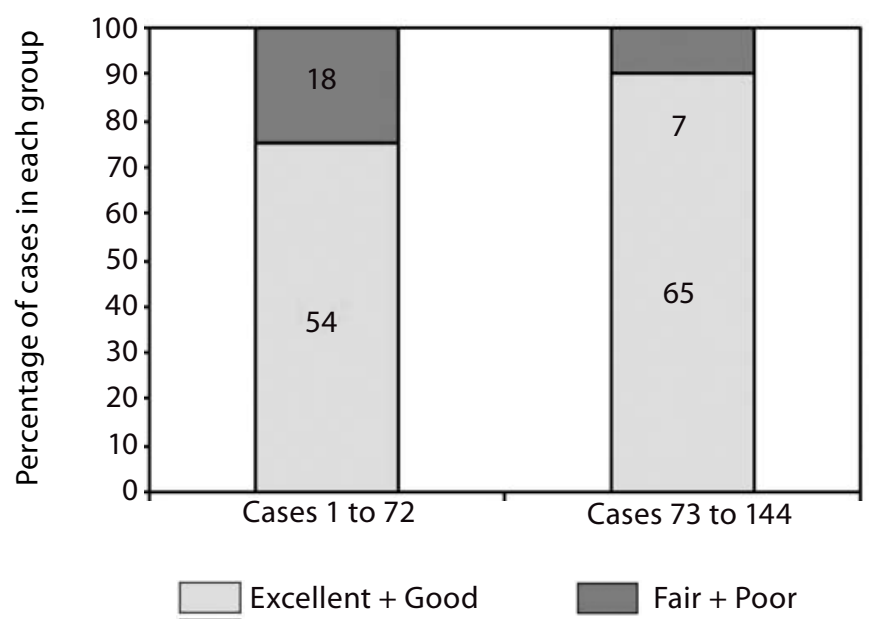

Distribution of results in the two groups.

\section{DISCUSSION}

Both groups had surgery under the same conditions, including the same operation room, equipment, and surgical instruments. Extruded herniations causing radiculopathy ${ }^{1,2}$ and radiculopathy at the L5-S1 level were not excluded, in contrast with previously published studies, ${ }^{6,9}$ that used first-generation endoscopes. If the disc herniations are stratified to separate contained herniations from extruded, sequestered herniations, the learning curve would be shortened to less than 15 procedures, and the success rate of the ideal herniations for the endoscopic approach would improve to $95 \%$.

The average ages of both groups are similar (Table 5). The factors that contributed to the decrease in operation time for group 2 were fewer multilevel interventions ( 17 in group 2 vs 36 in group 1) and a lower disc-case rate (1.27 discs/case in group 2 vs 1.55 in group 1). This time reduction and the improvement in the overall results from group 1 to group 2 are probably the result of a more accurate diagnostic recognition of endoscopic pathoanatomy and the surgeon's technical improvement. This improvement is multifactorial, ranging from accepting only the best skin portal and approach trajectory to the herniation to knowing when the herniation is appropriately decompressed (surrounding tissue pulsation) without having to visualize the exiting and traversing nerve. When needed, the traversing and exiting nerve can be visualized, but visualizing the traversing nerve may create additional morbidity by directly entering the epidural space.

\section{Table 5}

Characteristics of Foraminal Endoscopic Discectomy Patients, by Group

\begin{tabular}{lcc} 
& $\begin{array}{c}\text { Group 1 } \\
\text { (Cases 1-72) }\end{array}$ & $\begin{array}{c}\text { Group 2 } \\
\text { (Cases 73-144) }\end{array}$ \\
\hline Mean age in years & 45.5 & 45.4 \\
Sex & & \\
Male & 45 & 51 \\
$\quad$ Female & 27 & 21 \\
Operated discs, no. & 112 & 92 \\
One-level surgery, no. & 36 & 55 \\
Two-level surgery, no. & 32 & 14 \\
Three-level surgery, no. & 4 & 3 \\
\end{tabular}

The average age for those with fair and poor results was 50 years, which is close to the average age overall. The 1.48 discs per case rate for the fair and poor results group was only slightly higher than the overall average of 1.41 discs per case.

The disc-level distribution for the group of fair and poor results was of $13.6 \%$ for L3-L4 (5 discs), 43.2\% for L4-L5 (16 discs), $43.2 \%$ for L5-S1 (16 discs), and $0 \%$ for L1-L2 and L2-L3. The percentage of L5-S1 operated discs in the group of fair and poor results is slightly higher $(5.5 \%)$ than in the overall disclevel statistics (Table 1), so L5-S1 cannot be considered a main cause of the poor and fair results.

As stated in Cook et al., "Case mix sometimes complicates assessment of learning curves because as the surgeon becomes more experienced, the cases attempted become more difficult." $11(\mathrm{p} 422)$ That also applies here; as the confidence of the surgeon handling this technique rose, cases that had been rejected earlier were included and solved.

The following factors, in our opinion, contribute to the improvement of results: 
- Less instrument manipulation during the procedure because of optimal needle placement; careful introduction of the dilator with greater attention to its location in the cranial and lateral foraminal region to avoid irritation of the dorsal root ganglion

- Reduction of all exploration movements of endoscopic instruments to the minimum amount needed

- Precise cannula configuration selection according to the anatomical region

- Better recognition of the pathoanatomy with identification of the damaged tissues; subsequent treatment of annular tears and inflammatory membrane with radiofrequency therapy

- Identification and cutting of the superior foraminal ligament, posterior longitudinal ligament, and annular tissue if necessary to access the epidural space; proper bone foraminoplasty in foraminal stenosis ${ }^{10,13}$

- Selective and direct approach for reaching the herniation; correction of the entry point and angle depending on location of herniation. Central herniation more lateral entry and horizontal needle direction; foraminal herniation: more medial entry and vertical needle direction

- Technical ability to access L5-S1 routinely by selecting ideal needle trajectory and performing a lateral facetectomy if needed

The learning curve is steep (see the slope ${ }^{14}$ of the learning curve in Figures 1 and 2) because of the complexity of the surgical endoscopic technique. Patient selection also improved as the surgeon became more experienced in correlating the interpretation of magnetic resonance imaging with the endoscopic visualized pathoanatomy such as annular tears, foraminal osteophytes, and lateral recess stenosis, which is easy to miss and difficult to approach with traditional transcanal surgery.

It took approximately 60 cases for a confident identification of the exiting and traversing nerve roots. The ability to do a proper foraminoplasty took more than 80 cases. The use of differently shaped and bevelled cannulas helped to explore the epidural space and the nerve roots. There was minimal danger of causing nerve injuries because of the approach through Kambin's safety triangle. ${ }^{6}$

The results after the learning curve (90\% of excellent/good results reached) were similar to the results reported by other authors with larger series of cases with endoscopic procedure (e.g., Yeung, 219 cases $^{2}$ and 500 cases $^{3}$ ).
The calculated learning curve, as seen in Figure 1, does not present an asymptote as required by Cook et al. ${ }^{11}$ For the asymptote the series of cases has to be averaged (see black line in Figure 2). The obtained asymptote of the averaged learning curve represents a good approximation of the rate of results that has been observed since completing the learning process.

This study's learning curve and results are based only on a single surgeon's experience. The results of others may vary depending on their training conditions and previous spine surgery experience. This is why, in order to broaden this study and find results each time closer to generalization, we encourage surgeons to use the methodology presented here to calculate their own learning curve and publish their results.

In summary, we studied 144 cases of endoscopic disc surgery and explored a method to calculate the surgeon's learning curve. The method to calculate the learning curve was based on an outcome algorithm and a surgical time evolution until both achieve an asymptote of consistent values. The learning curve was established after the first 72 cases. After the learning curve experience was complete, this surgeon reached and surpassed a success rate of $90 \%$ for this endoscopic surgical technique. The method was applied to a single surgeon's experience; the learning curve results for this endoscopic surgical technique may vary for other surgeons.

Rudolf Morgenstern, MD, PhD, Christian Morgenstern, Dipl-Ing, and Anthony T. Yeung, MD

From Centro Médico Teknon, Barcelona, Spain (R. Morgenstern); Universitat Politècnica de Catalunya, Barcelona, Spain (C. Morgenstern); and Squaw Peak Surgical Facility, Phoenix, Arizona (Yeung).

No financial aid or funding was received for this study. There was no possible conflict of interest.

Address correspondence and reprint requests to Rudolf Morgenstern, c/o Pedro y Pons 18-20, E-08034 Barcelona, Spain (email: rumor@ endoscopiacolumna.com).

This submission was received March 20, 2007, and accepted for publication June 1, 2007

Because this study was a case outcome study, no protocol approval was needed.

\section{REFERENCES}

1. Yeung AT, Tsou PM. Posterolateral endoscopic excision for lumbar disc herniation: surgical technique, outcome, and complications in 307 consecutive cases. Spine. 2002;27:722-731.

2. Tsou PM, Yeung AT. Transforaminal endoscopic decompression for radiculopathy secondary to intracanal noncontained lumbar disc herniations: outcome and technique. Spine J. 2002;2:41-48.

3. Yeung AT. The evolution of percutaneous spinal endoscopy and discectomy: state of the art. Mt Sinai J Med. 2000;67:327-332. 
4. Hermantin FU, Peters T, Quartararo L, Kambin P. A prospective, randomized study comparing the results of open discectomy with those of video-assisted arthroscopic microdiscectomy. J Bone Joint Surg Am. 1999;81:958-965.

5. Hijikata S. Percutaneous nucleotomy. A new concept technique and 12 years' experience. Clin Orthop Relat Res. 1989;(238):9-23.

6. Kambin P, Gellman H. Percutaneous lateral discectomy of the lumbar spine: a preliminary report. Clin Orthop Relat Res. 1983;174:127-132.

7. Forst R, Hausmann G. Nucleoscopy: a new examination technique. Arch Orthop Traum Surg. 1983;101:219-221.

8. Schreiber A, Suezawa Y, Leu H. Does percutaneous nucleotomy with discoscopy replace conventional discectomy? Eight years of experience and results in treatment of herniated lumbar disc. Clin Orthop Relat Res. $1989 ;(238): 35-42$.

9. Mathews HH. Transforaminal endoscopic microdiscectomy. Neurosurg Clin N Am. 1996;7:59-63.

10. Knight MTN, Goswami AKD. Endoscopic laser foraminoplasty. In: Savitz MH, Chiu JC, Yeung AT, eds. The Practice of Minimally Invasive Spinal Technique. 1st ed. Richmond, Virginia: AAMISMS Education LLC; 2000:337-340.

11. Cook JA, Ramsay CR, Fayers P. Statistical evaluation of learning curve effects in surgical trials. Clin Trials. 2004;1:421-427.

12. Morgenstern R, Morgenstern C, Abelló A, Vilella C. SchifferdeckerHoch F. Eine Studie von 144 Fällen nach unterzogener endoskopischer Lendenwirbelsäulenchirurgie. Klassische Rehabilitation im Vergleich zur FPZ Methode. Orthopädische Praxis. 2005;12:674-681.

13. Yeung AT, Yeung CA. Advances in endoscopic disc and spine surgery: foraminal approach. Surg Technol Int. 2003;11:253-261.

14. Buchmann P, Steurer J. The learning curve in surgery: possibilities and limits of this method [in German]. Swiss Surg. 2002;8(3):106-109. 